\title{
Face-on and Edge-on Impact Response of Composite Laminates
}

\author{
Wooseok $\mathrm{Ji}^{1}$ \\ Ulsan National Institue of Sciece and Technology, Ulsan, Korea \\ Sunil Pai Sringeri ${ }^{2}$, Solver Thorsson ${ }^{3}$, Cyrus Kosztowny ${ }^{4}$ and Anthony M. Waas ${ }^{5}$ \\ University of Michigan, Ann Arbor, 48105 \\ and \\ Mostafa Rassaian ${ }^{6}$ and Salvatore Liguore ${ }^{7}$ \\ Boeing Company, Seattle, Washington, 98124
}

\begin{abstract}
This paper presents comprehensive experimental and numerical studies on the face-on and edge-on impact behavior of composite laminates. Experimental work is focused on finding the impact energy limits for barely visible impact damage (BVID) when a laminated composite plate is impacted either on its face or an edge. High-fidelity finite element analysis (FEA) model utilizing Enhanced Schapery theory (EST) combined with Discrete Cohesive Zone Model (DCZM) is developed to predict the response of the laminated composite plate subjected to face-on and edge-on impact loading. Predictions from the proposed high-fidelity FEA model are compared against experimental data and it is shown that the numerical results agree well with the test data.
\end{abstract}

\section{Introduction}

$\mathrm{C}$ OMPOSITE laminates are increasingly being used in aero-structural applications where weight reduction is needed. When laminated composites are used in structural applications, structural integrity and damage tolerance (SIDT) limits are used in the design process. Laminated composite structures, in service, can be subjected to lowenergy impacts, which may leave barely visible signs on the impacted surface but a question of interest is the extent of damage through the thickness. Barely visible damage caused by impact events can reduce the strength and stiffness of laminated composite structures ${ }^{1}$.

Many research studies have been done to define the barely visible impact damage (BVID) limit of composite structures that occurs at low-energy levels. Even by low-velocity impact loading, composite structures exhibits very complicated and interactive failure mechanisms, resulting from multiple failure modes such as fiber failure, matrix micro cracking and delamination that can be initiated either simultaneously or sequentially. Characterization of the response of composite structures to suddenly applied loads is not an easy task to be done, both experimentally and theoretically.

The research presented here includes both experimental investigations and finite element simulations of impact damage on a laminated composite plate. The overall aim of this work is to develop an understanding of impact behavior of a composite plate when an object hits its face and/or its edge. BVID limits for the two loading scenarios will be determined from experimental examination and high-fidelity finite element analysis (FEA) is performed to highlight detailed mechanisms of damage progression due to face-on and edge-on impact loading.

\footnotetext{
${ }^{1}$ Assistant Professor, School of Mechanical and Nuclear Engineering, 301-3 $1^{\text {st }}$ Engr. Bldg., AIAA Member

${ }^{2}$ Graduate Student Research Assistant, Department of Aerospace Engineering, B203 FXB

${ }^{3}$ Graduate Student Research Assistant, Department of Aerospace Engineering, B203 FXB

${ }^{4}$ Graduate Student Research Assistant, Department of Aerospace Engineering, 2045 FXB

${ }^{5}$ Felix Pawlowski Collegiate Professor, Department of Aerospace Engineering, 3044 FXB, AIAA Fellow

${ }^{6}$ Technical Fellow, Boeing Research and Technology, Seattle, WA, AIAA Associate Fellow

7 Technology Manager Boeing Research and Technology, AIAA Sr. Member
} 


\section{Face-on and Edge-on Impact Tests for BVID}

\section{A. Test Setup}

Drop-weight testing apparatus used for the impact experiments is shown in Figure 1. It consists of a steel support structure constructed using I-beams. The impact fixture is placed on a solid steel pedestal so as to a provide a completely flat and rigid surface. The impactor assembly consists of a dead-weight (a solid steel block in this case), the lower end of which is attached to a threaded metal rod into which the impactor is bolted. The other end of the impactor assembly is connected to a steel rope which, in turn is connected to a pulley. The impact assembly can be raised or lowered by rotating the handle connected to the pulley. Low-friction bearings attached to the ends of the dead-weight are responsible for the translational movement of the impact assembly through lubricated guide rods which run the length of the drop tower and are firmly affixed to the support structure. A force sensor is screwed onto the threaded cylinder and sits between the impactor and the dead weight. The impact energy isdirectly proportional to the height from, which the impact assembly is dropped on to the specimen housed in the fixture. A zip-tie made from high strength plastic is used to attach the impact assembly to the looped end of the steel rope. Based on the required energy level, the pulley is rotated, thereby raising the impact assembly. The taut zip-tie is now snapped in a swift, clean stroke resulting in a free-fall impact onto the specimen.

A schematic shown in Figure 2 illustrates the entire impact test setup with data acquisition (DAQ) system. The force sensor picks up the load from the impact and this signal is sent through an amplifier and recorded on an oscilloscope. Simultaneously, a TTL trigger signal is sent to a stereo setup of high speed cameras running at 50,000 frames per second. The stereo setup is utilized so as to obtain a 3D digital strain field map. The event is captured by the cameras, and the required number of frames are saved. Digital image correlation (DIC) techniques using a commercial software, ARAMIS, is then utilized to calculate the full-field strains and displacements.

\section{B. Experiment Resutls}

Various impact energies were used to identify the BVID limit. It was clear that the lower energies were the ones of interest. Therefore, the energy levels to be examined were set to be low values of several joules. Figure 3 shows the maximum out-of-plane displacement of the composite plate that is subjected to face-on impact loading. The displacement is normalized by the composite plate thickness. Figure 4 shows the load signals for each of the 5 energy levels, from the time the impactor hits the specimen and then rebounds off. The 5 energy levels are normalized with the impact energy at the BVID limit. The results reported here were examined for repeatability. Figure 5 shows the average indent depths for each of the energy levels. The indent depth was measured using a dial indicator. It can be noted that the results are fairly linear.

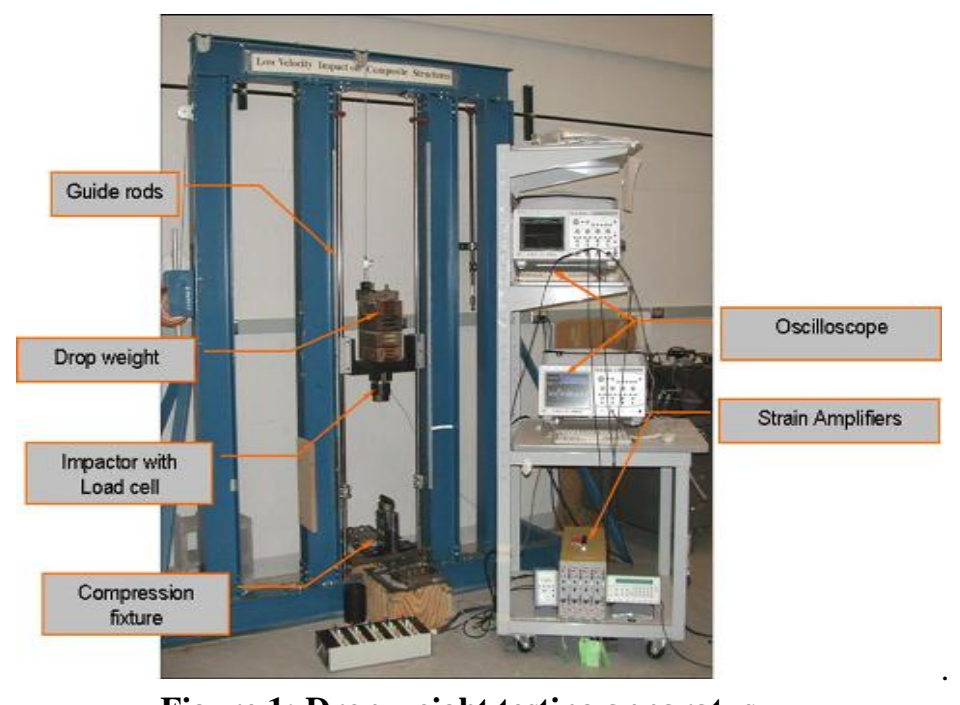

Figure 1: Drop weight testing apparatus 


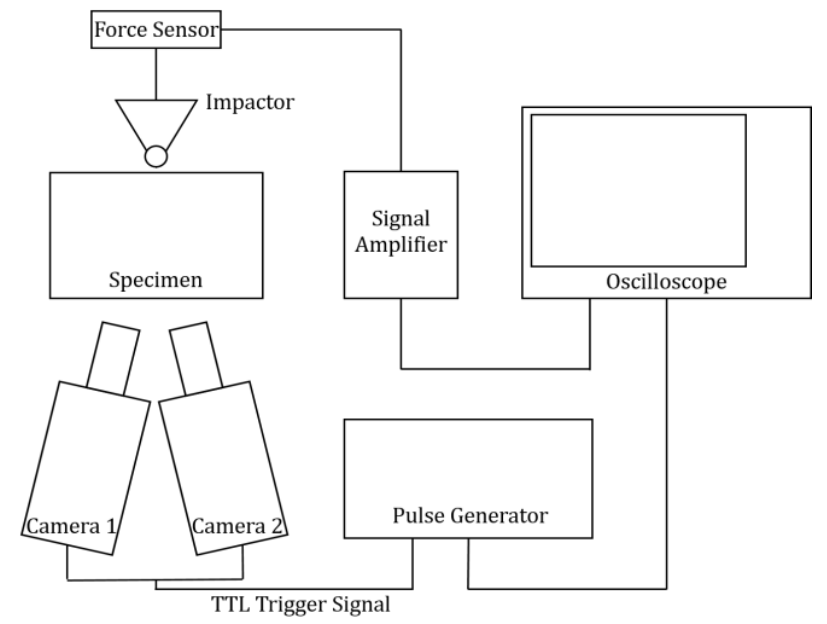

Figure 2: Schematics of data acquisition system

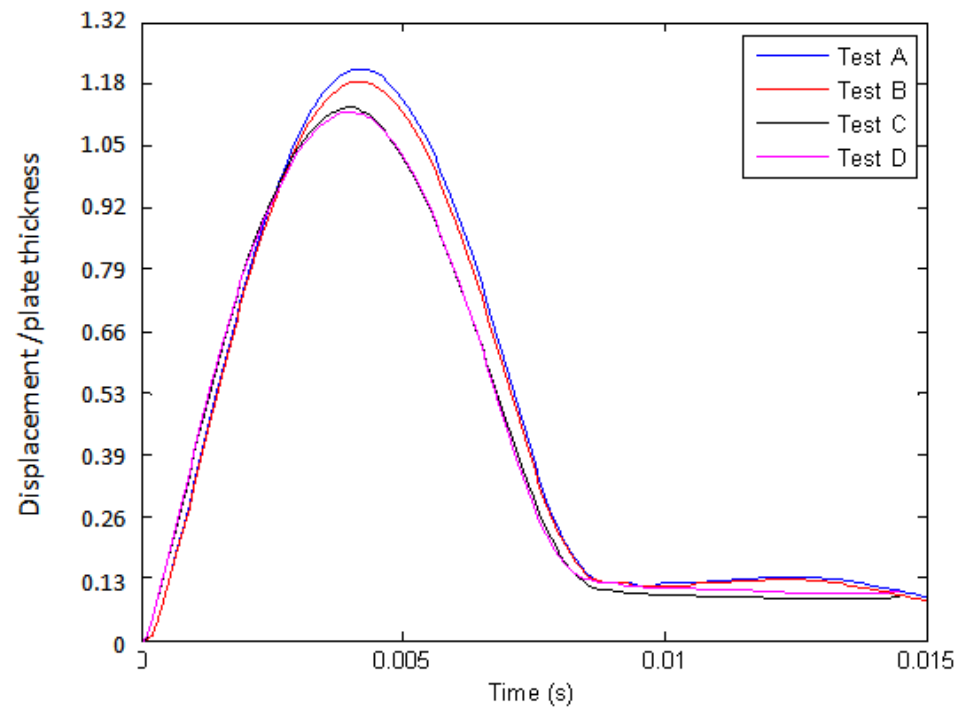

Figure 3: Maximum displacement response for face-on impact 


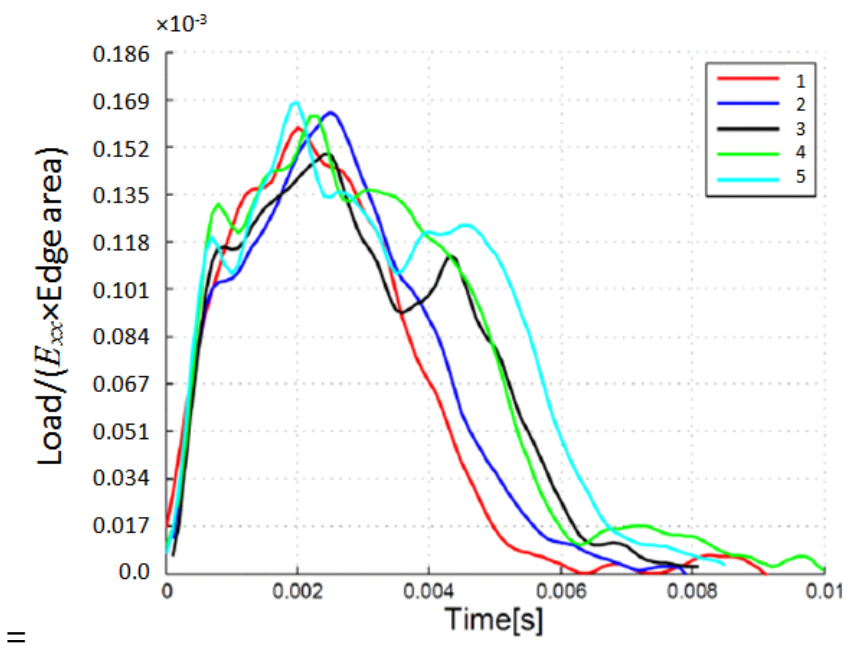

Figure 4: Impact load signals of each energy levels for the case of edge-on impact

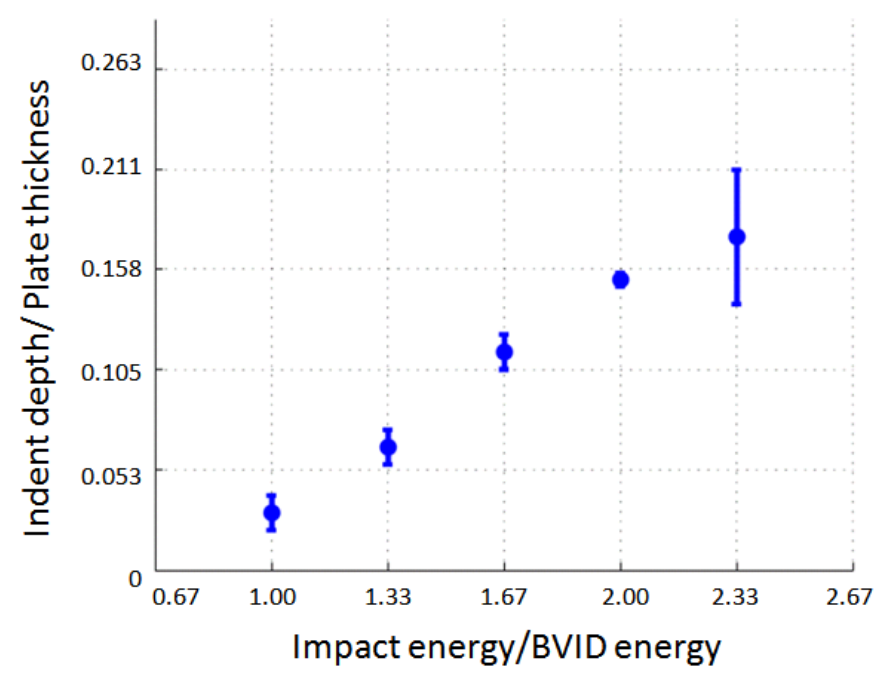

Figure 5: Average indent depth for each of edge-on impact

\section{High-Fidelity Finite Element Analysis Model}

The response of a composite plate that is subjected to impact loading is very complex and complicated, and thus a finite element-based analysis is a viable tool to capture details of the impact response that occurs within very short time duration. A progressive damage and failure analysis model is developed by utilizing Enhanced Schapery theory $(E S T)^{2}$ for in-plane damage and failure, combined with Discrete Cohesive Zone Model (DCZM) ${ }^{3}$ for out-of-plane failure.

\section{A. Enhanced Schapery theory}

EST is capable of modeling the constitutive behavior of fiber-reinforced composite laminates over the full spectrum including damage and failure. Here, damage is considered as a mechanism that is responsible for material nonlinearity before the material reaches a critical state. Failure is defined as the post-peak softening behavior until the material completely loses stiffnesses. EST combines Schapery theory ${ }^{4}$ for modeling damage with Crack Band model ${ }^{5}$ for predicting failure. Schapery theory (ST) has been proven to be an accurate and effective homogenized model for progressive damage analysis of laminated composites that exhibit nonlinear behavior due to matrix microcracking ${ }^{6,7}$. Schapery theory decomposes the total energy into the dissipated energy, $S$, due to matrix microcracking and the

American Institute of Aeronautics and Astronautics 
recoverable elastic strain energy ${ }^{2}, W_{E}$. As the damage is accumulated, the material strength is degraded until failure initiates. After failure initiates at the peak load, the Bazant-Oh crack band model ${ }^{5}$, modified to be applied to laminated composites as described in Ref. 2, computes the release of the fracture energy, $G_{C}$, over a characteristic dimension, $L_{\mathrm{el}}$. Figure 6 schematically describes EST that combines ST and Crack Band model in order to describe the material behavior over the full range.

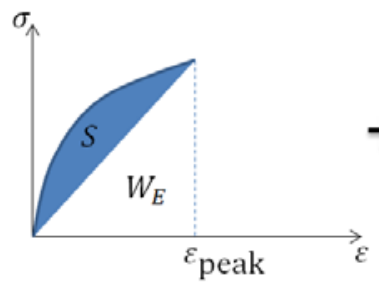

(a) Schapery theory

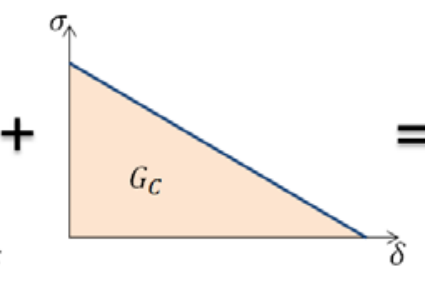

(b) Crack Band

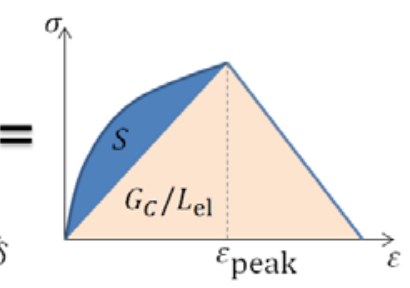

(c) Enhanced ST

Figure 6: Theoretical background of the enhanced Schapery theory (EST)

\section{B. Face-on Impact Finite Element Analysis}

A computational model was generated, having dimensions matching the physical impact specimens of $150 \mathrm{~mm}$ by $100 \mathrm{~mm}$. Simple supports were used on all four edges, and a 1 inch rigid impactor set to a 20 Joule impact was also modeled. Each lamina was modeled using S4 elements, and each lamina uses Schapery theory to capture in-plane material damage. Discrete Cohesive Zone Modeling (DCZM) elements were used in-between each lamina to capture delamination behavior. All lamina and DCZM properties used were provided by Boeing. Figure 7 shows specific layer-by-layer information from the Schapery theory damage and delamination area using quasi-static properties as provided by Boeing. Note that the material degradation (first row of images) is provided by Schapery modeling, and the delamination (next two rows) is from DCZM modeling.

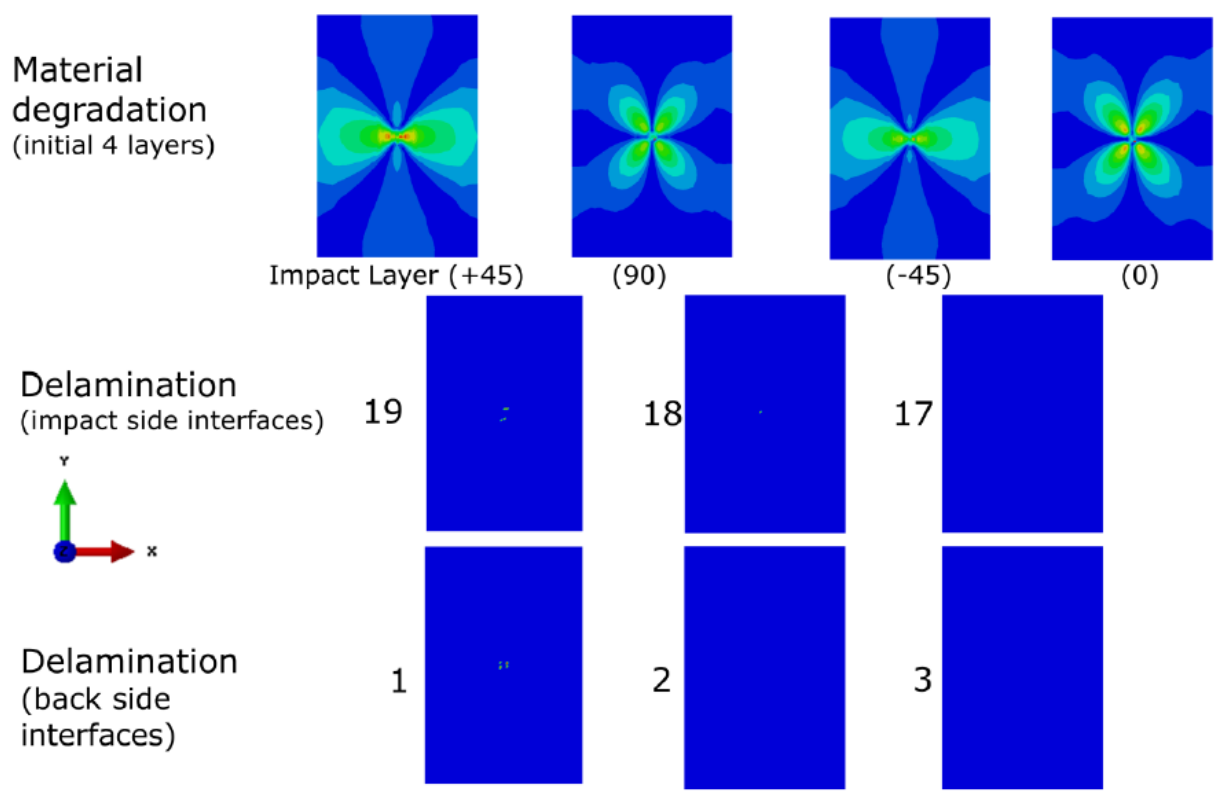

Figure 7: Damage and delamination behavior predicted by Schapery theory and DCZM. Note that interface 19 is between the 1st and 2nd lamina on the impact surface.

From Figure 7, the predicted delamination area is seen to be significantly small. As a result, a parameter study on the cohesive strengths and fracture toughnesses was conducted. The cohesive strengths showed slight alteration of the delaminated area. Decreasing the values removed all delaminations, and increasing the values led to slightly increased delamination area. On the other hand, fracture toughnesses, however, showed significant influence on the delamination area. Decreasing fracture toughness, significantly increases the layer-by-layer delamination area as well as the 
through-the-thickness extent of delamination. Figure 8 shows the Schapery material degradation and delamination behavior for reduced fracture toughness (and as provided cohesive strengths). This result stems from the triangle traction separation law used within the DCZM elements. As the cohesive strengths are held constant, decreasing the fracture toughness results in less area under the triangle. With less area under the triangle, the elements have less displacement to undergo before fully traveling along the separation law and failing. The overall out-of-plane displacement and load history trends were not significantly affected by altering the cohesive strengths or fracture toughnesses.

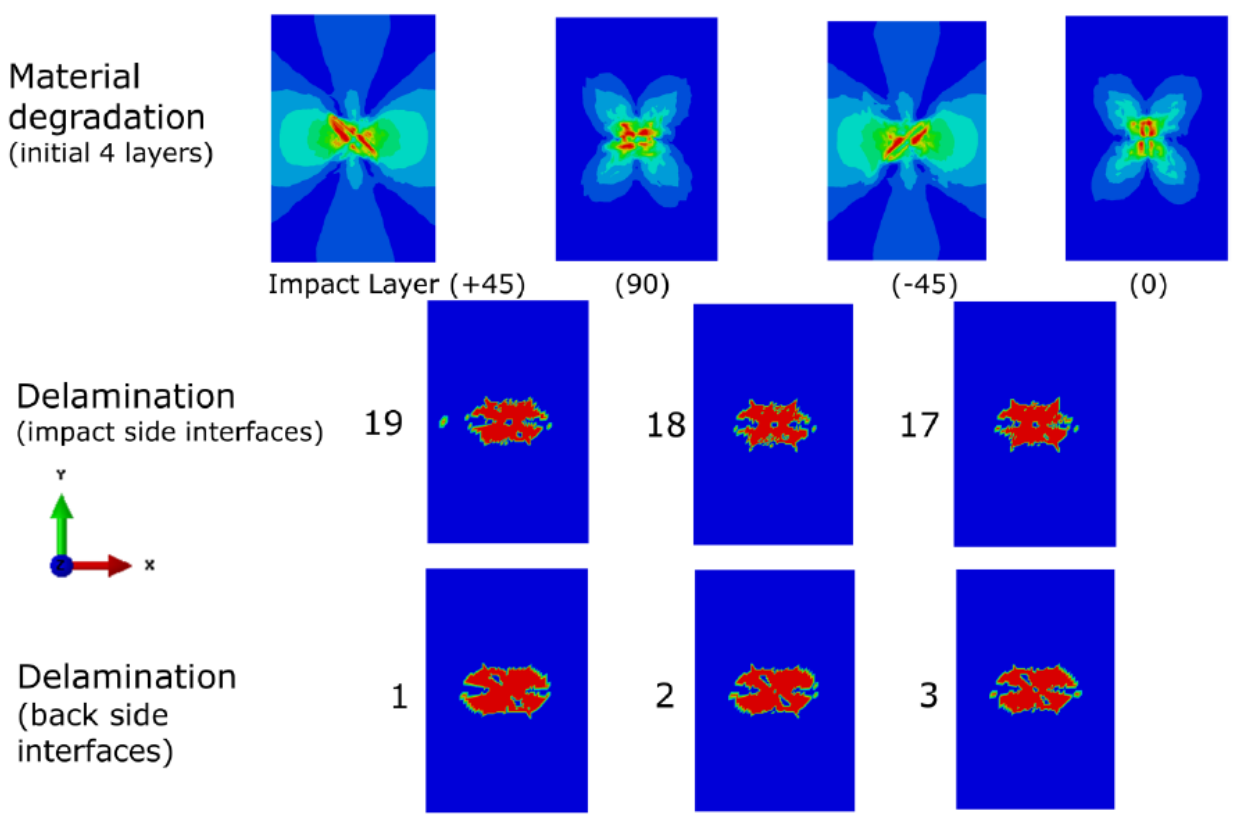

Figure 8: Increased delamination areas as a result of decreased fracture toughness.

\section{Edge-on Impact Finite Element Analysis}

Figure 9(a) shows the impact test fixture with the specimen mounted on the fixture. Figure 9(b) illustrates its representative finite element model composed of the composite plate and the impactor. The boundary conditions used in the model are also indicated in Figure 9(b). The laminated composite plate is modeled as a single shell layer. Since the focus of the analysis has been limited to lower energy levels to define the BVID limit, the single shell layer is sufficient to predict the impact response when delamination is of no interest. The impactor is modeled with rigid body elements, R3D4 available in ABAQUS. The finite element meshes of the two objects are shown in Figure 8. For the coupon, very fine meshes are used near the impact point and coarse meshes are used for the regions that are hardly affected by the impactor. Since the response to the edge-on impact is highly localized near the contact point, EST is applied in the specific region and an elastic material model is used elsewhere, as indicated in Figure 10, respectively. FE meshes of the impactor are also shown in Figure 10.

Buckling analysis is first performed to obtain the shape of an initial geometric imperfection. The composite plate in reality is not perfectly flat and straight, and the effect of unintended initial geometric imperfections on the impact response should be accounted for when the impact analysis is performed. Figure 11 shows the finite element model for the buckling analysis, which is used to generate buckling mode shapes which are subsequently used to generate an initially crooked plate. The same boundary conditions as the subsequent impact analysis is enforced on the plate, but the non-uniform pressure as shown in Figure 11 is applied on the top edge in replacement of the impactor. Note that this is done only for the buckling analysis that produces a mode shape and not for subsequent impact analysis. Figure 11 also shows the first buckling mode shape, which is used to seed the initial geometric imperfection to the impact analysis model. 


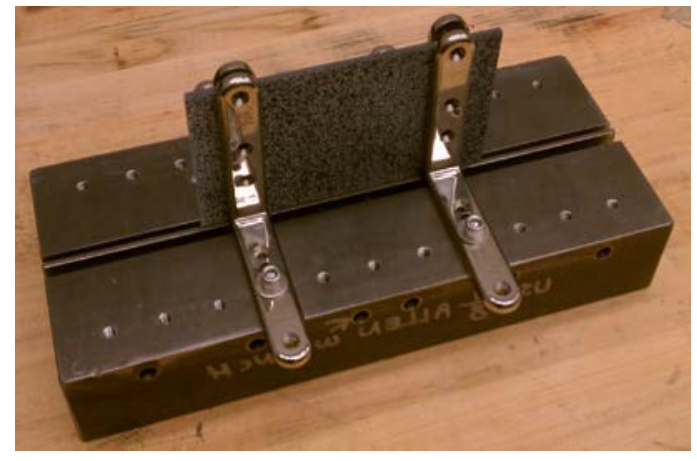

(a)

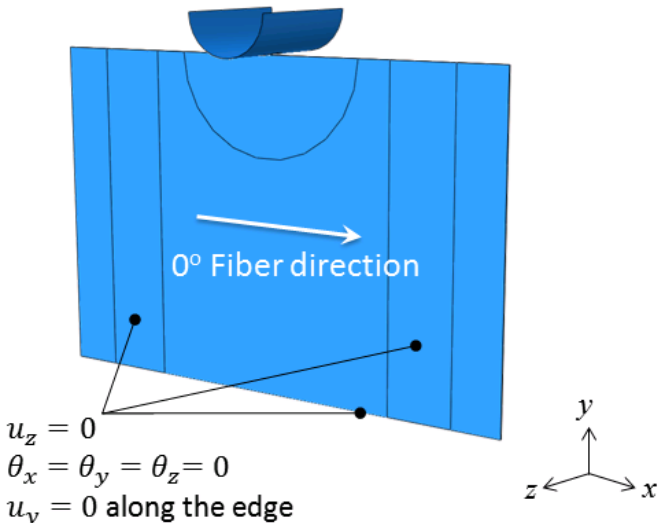

(b)

Figure 9: (a) Edge-on impact test fixture (b) Finite element model of the impact simulation

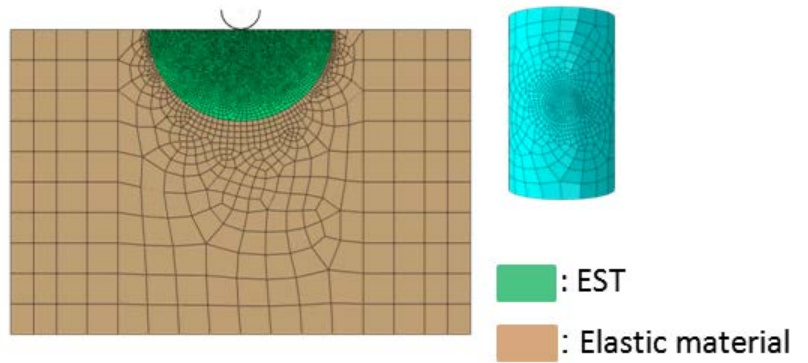

Figure 10: Finite element meshes of the plate and impactor
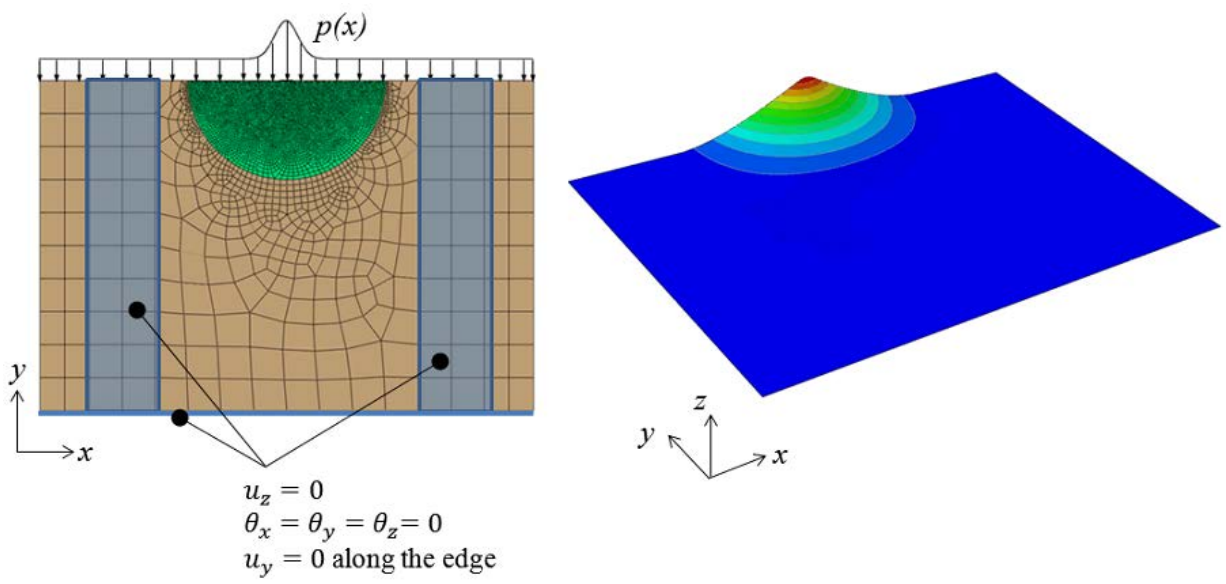

Figure 11: Finite element model for buckling analysis and the first buckling mode shape

The impact simulation is performed using ABAQUS/Explicit with EST implemented via user material subroutine. The plate is modeled with S4R shell elements and the impactor is modeled with R3D4 rigid elements. All the simulation results in this paper are limited to the BVID energy, identified from experiments. Figure 12 and 13 show the impact response of the plate from various views. The deformed configuration of the plate is captured after the impactor is first rebounded. Shell elements assume that the deformation in the through-the-thickness direction conforms to that of the mid-plane, and therefore the effect of the through-the-thickness deformation is hardly captured. As a result, the detailed deformation at the impacted edge may not be accurate. However, the global response from the simulation is captured very well from the model as shown in Figure 14. As shown in Figure 12, the damage variable, $S_{r}$, is also examined to visualize the extent of damage due to the impact loading. 

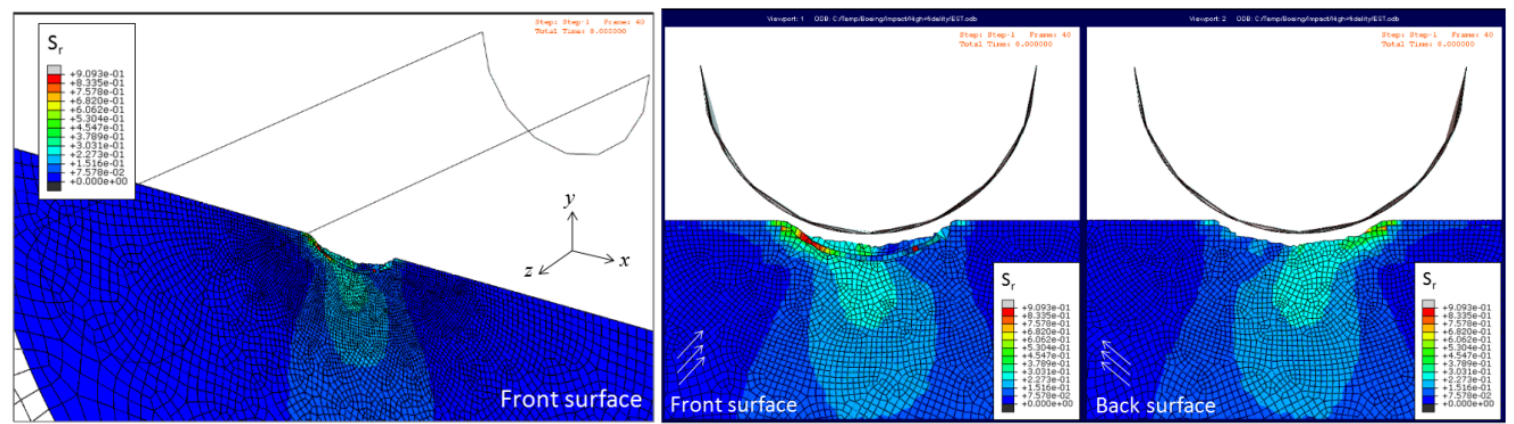

Figure 12: Deformed configuration of the plate after the impactor is rebounded.

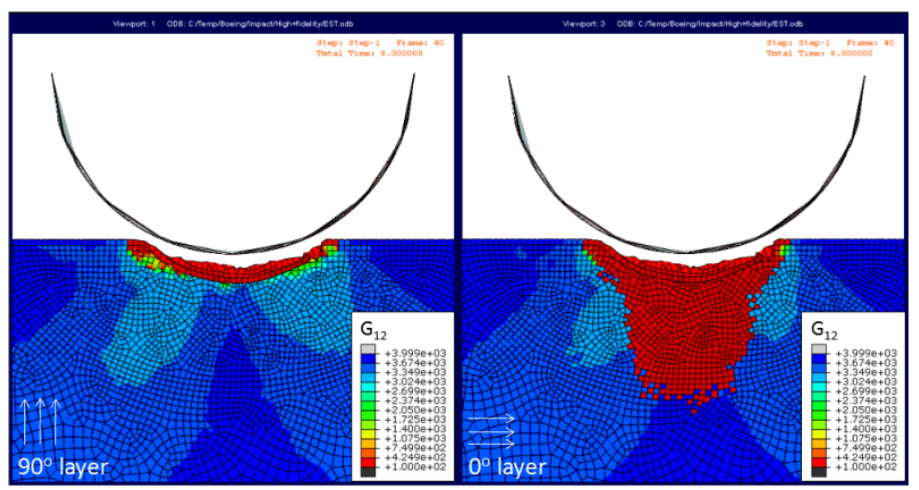

Figure 13: Degraded shear stiffness value at the 2 nd $\left(90^{\circ}\right)$ and 4 th $\left(0^{\circ}\right)$ layers

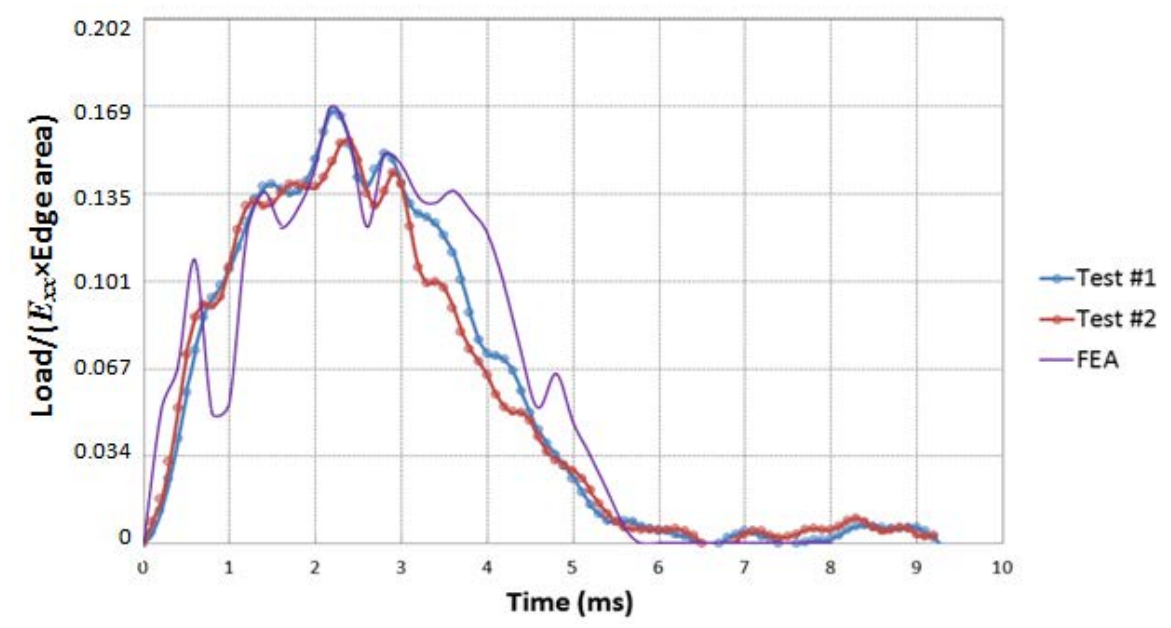

Figure 14: Comparison of load-time history obtained from experiments and simulation

The effect of the geometric imperfection is studied and the results are summarized in Figure 15. The effect of the geometric imperfection is parameterized with the maximum amplitude of the imperfection shape. As shown in Figure 15, different values of the amplitude have minimal effects on the impact response. Figure 16 shows that the impact response is more sensitive to the residual strength of the damaging material. It is necessary to define the residual strength of the material since the detailed failure mechanism is extremely complicated at the impacted region. Right under the impactor, fibers are prone to break, but broken fibers are compacted together and create a newly stiffened region with the chopped fibers. Since EST is developed in the macro-length scale by homogenizing fibers and matrix, the aforementioned fiber failure mechanism cannot be accurately captured. Instead, its effect can be estimated by setting the minimum residual strength of a lamina. 


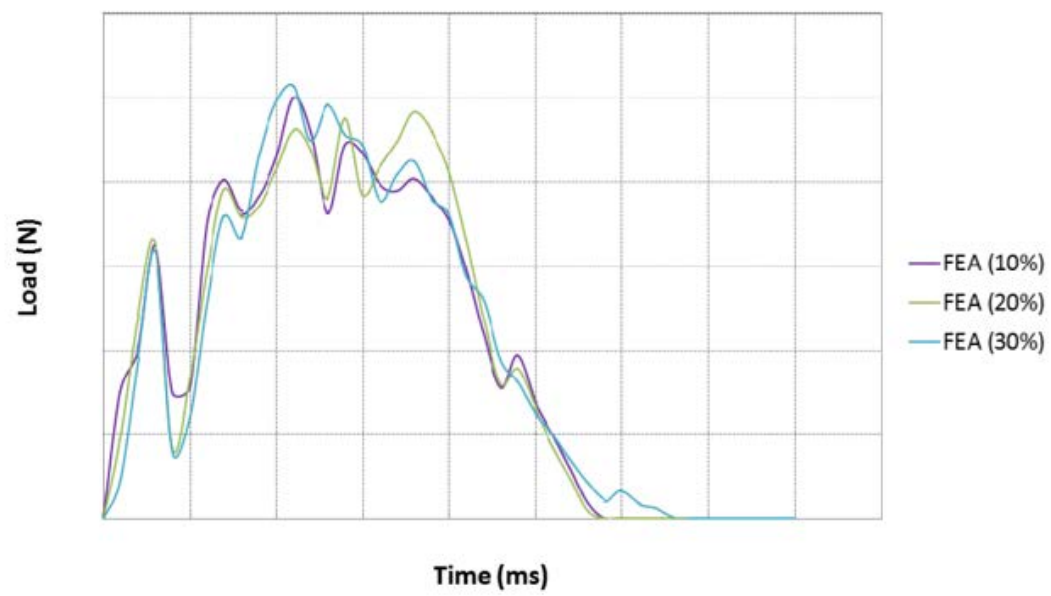

Figure 15: Effect of the geometric imperfection as a function of the amplitude

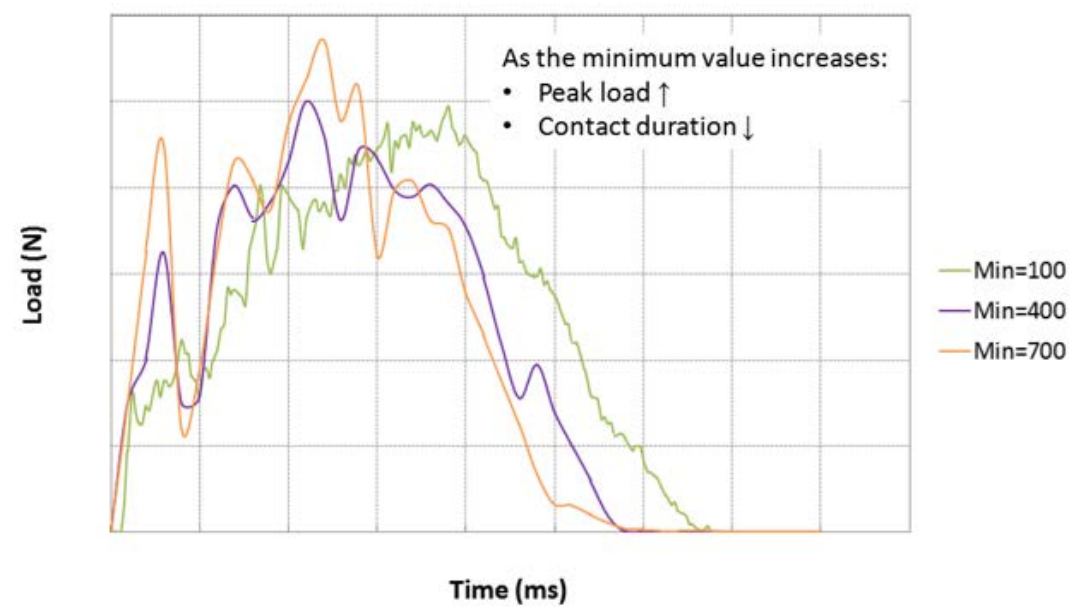

Figure 16: Effect of the residual strength on the impact response

\section{Conclusions}

Results from a comprehensive experimental and numerical study on the BVID limits of a composite plate subjected to face-on and edge-on impact loading has been presented. BVID limits for the two loading cases were found from the experimental work utilizing the drop-weight testing apparatus. Detailed characteristics of the damage at the BVID limits were also obtained from the experiments. High-fidelity finite element analysis is performed to shed light on the response of structure that is loaded over a very short time period. Due to the omnipresence of multiple failure modes, a unified numerical model that is capable of predicting in-plane and out-of-plane damage and failure modes was employed. The simulation results performed at the BVID limits provided information about the impact events in full detail and agreed very well with the experimental observation at low-velocity impact.

\section{Acknowledgments}

The authors are grateful for financial support from the Boeing Company.

\section{References}

${ }^{1}$ Abrate, S., “Impact on laminated composite materials,” Journal of Applied Mechanics, Vol. 44, No. 4, 1991.

${ }^{2}$ Pineda, E.J. and Waas, A.M., "Numerical implementation of a multiple-ISV thermodynamically-based work potential theory for modeling progressive damage and failure in fiber-reinforced laminates,” International Journal of Fracture, Vol. 182, No. 1, 2013, pp. 93-122.

${ }^{3}$ Schapery, R.A., "A theory of mechanical behaviour of elastic media with growing damage and other changes in structure," Journal of Mechanics and Physics of Solids, Vol. 38, No. 2, 1990, pp. 215-253.

American Institute of Aeronautics and Astronautics 
${ }^{4}$ Gustafson, P.A. and Waas, A.M., "Efficient and robust traction laws for the modeling of adhesively bonded joints," AIAA/ASME/ASCE/AHS/ASC 49th Structures, Structural Dynamics, and Materials Conference, CP2008-1847, Schaumburg, IL.

${ }^{5}$ Bazant, Z.P. and Oh, B.H., “Crack band theory for fracture of concrete,” Materiaux et constructions, Vol. 16, No. 93, pp. 155177.

${ }^{6}$ Basu, S., Waas, A.M. and Ambur, D.R., “Compressive failure of fiber composites under multi-axial loading,” Journal of the Mechanics and Physics of Solids, Vol. 54, 2006, pp. 611-634.

${ }^{7} \mathrm{Ji}, \mathrm{W}$. and Waas, A.M., "Progressive failure analysis for the interaction of interlaminar and intralaminar failure modes in composite structures with an initial delamination,” The Aeronautical Journal, Vol. 117, No. 1187, 2013, pp. 71-84. 